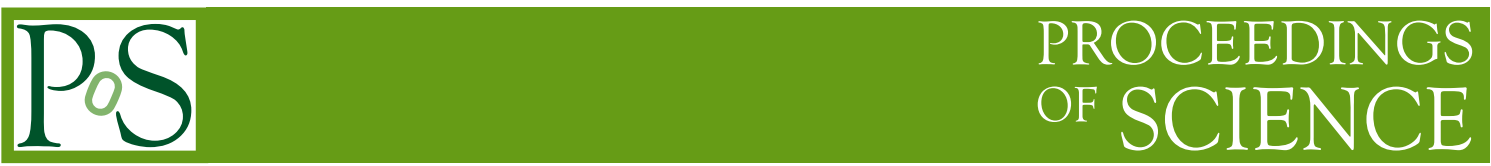

\title{
No magic wand for teaching physics
}

\section{Damir Buskulic*, Gilles Maurin}

LAPP, Université Savoie Mont Blanc, CNRS/IN2P3, Annecy-le-Vieux, France

E-mail: buskulicelapp.in2p3. fr

\section{Richard Taillet}

LAPTh, univ. Savoie Mont Blanc, CNRS, BP 110, Annecy-le-Vieux F-74941, France

\begin{abstract}
Teaching physics is about pedagogy, didactics, but mainly physics. The university physics teacher, being most of the time also a researcher, devotes a large fraction of his time to preparation of courses, exercises, lab work. The pedagogical questions are often felt as the realm of specialists, or too time consuming, leading to the easy option of leaving those questions implicit. Is it possible to improve our teaching without becoming experts in didactics ? At the physics group of the Université de Savoie, we experimented a few techniques and options, with the help of experts in pedagogy. Among others, we will describe the use of the online teaching platform WIMS that allows random exercises, has a large choice of interaction methods and a formal engine; the use of videos of physics courses to be viewed online; experiments in tutorial classes; the use of audience response devices (clickers), in connection to active learning. We are also experimenting some efficiency measuring tools (concept inventories). Finally, we started recently experimenting a flipped classroom. We hope that each and everyone of our colleagues will find a set of tools that suits his/her sensitivity.
\end{abstract}

Frontiers of Fundamental Physics 14

15-18 July 2014

Aix Marseille University (AMU) Saint-Charles Campus, Marseille, France

\footnotetext{
* Speaker.
} 


\section{Introduction}

Todays social and organisational changes impact the way we teach, and we will focus in this paper on science education in physics at the university level. The social changes are, among others, technological (computers, smartphones, the internet,...) and communicational (SMS, social web,...). On the organisational side, one can refer as an example to the Licence/Master's/Doctorate (LMD) system in Europe which was set up more than ten years ago. One of the indirect consequences is that the spread in diversity of the initial scientific background of the students joining the University has become greater, due to the diversity of their prior scholar path.

Considering this, one may ask if the teachers can keep following the traditional lecturing/training system. There is an actual need for a change in the most common pedagogy. One very clear fact is that many of us, teachers and professors, are primarily physicists, not researchers in pedagogy, albeit with a lot of good will.

\section{Innovative teaching methods}

In the past years, a lot of theoretical and practical tools were developped and tested around the world. Examples are audience response devices (also known as "clickers"), online learning platforms, peer instruction [2], flipped learning, hybrid teaching, interactive learning strategies, constructive alignment. All of these are placed in the context of "active" pedagogies. But the problem a non expert in pedagogy is confronted with is the choice of tools or strategies that best suit her/his pedagogical skills or knowledge. And such questions arise as should we work in a team or can these be used alone in the context of a class ?

John Biggs described three levels of teaching [1]. In the first level, the teacher distinguishes students as "good" and "bad", focusing on what he thinks students are. This is a teacher-centric way of teaching. In the second level, which is still teacher-centric, the teacher focuses on what he does, how he acts, having as a preconception that a good class makes students understand. The third level is student-centric and the emphasis is placed on the students actions and what they do. The idea is that what students do should match the intended learning outcomes of the class and of the assessments.

Marcel Lebrun from UCLouvain highlights the importance of the diversity of methods and tools available to the teacher. We believe this is of highest importance to achieve as much as possible the third level described above. We believe as well that there are no universal tools or methods and there is a need for tools adapted to each specific course and/or teacher.

\section{Diversity, a must : examples}

Located in Chamberry and Annecy in the French alps, our university, with 13000 students, is average sized. The number of students attending the physics classes is of the order of 150 for the first year, 120 in the second year, 20 in the third year, 20 at the Master's level and 10 to 15 preparing a PhD thesis. The physics department staff consists in 20 "enseignants-chercheurs" (with a status of teachers and at the same time researchers) and 50 researchers in 3 laboratories.

We started to work in many directions, testing many different tools that seem to be adapted to each of our personal pedagogical goals, either implicit or explicit. Several of us tried and tested the 
production and use of videos of physics courses, the production and use of online interactive exercises, tutorials in small groups, the use of audience response devices, assessment of learning gains via concept inventories, and recently flipped classrooms. In parallel, a pedagogical and technical coordination group was formed.

\subsection{Enhanced videos of physics courses}

One of us (R. Taillet) produced approximately 150 hours of videos [4] of physics lessons, in french. The videos span nine different courses in mechanics, electromagnetism, geometrical optics, physical optics, introduction to Special Relativity, introduction to General Relativity, history of Science, thermodynamics. A small contribution of a few hours was made by D. Buskulic in electrostatics and magnetostatics.

These videos were quite a big success, achieving 180000 complete downloads in two years, as well as 800000 downloads of the selection that was put on a popular video streaming service. They were used for exam preparation, discovery (think of pensioners, adults returning to university, curiosity), as a complement to other courses or late arrival during the year.

The production cost of these videos is quite high, representing 300 to 400 man-hours of work.

\subsection{Tutorials in small groups}

On another front, we experimented tutorials in small groups (G. Maurin). These were organized in elementary mechanics and geometrical optics. Instead of having a teacher (and sometimes a student) presenting the solution to a problem in front of a large group, the students are divided in small groups of 4 or 5 students, working as a team to solve a problem on a dedicated blackboard. Each student in turn has a role: scribe, writer on the blackboard or just participant to the reflexion. The teacher has the role of a "guide on the side".

The experiment was pursued at the first and third year of Licence, as well as at the first year of Master's. Since the results were quite positive, the teacher involved decided to turn this experiment into a regular practice.

The students who were new to the university system (first year of Licence) and those who were mature enough (first year of Master) were enthusiastic, which is a success by itself. There were more problems with students from third year of Licence, who did not know how to get organized. We interpreted this as the effect of their past experience. They never had anything else than the "standard" system.

\subsection{Peer instruction and Audience Response Devices}

Linked to "peer instruction" pioneered by E. Mazur in the 90's [2][3], a few teachers in our university experimented and implemented the use of audience response devices. For each lesson, there are interleaved sections where the instructor first makes a 10-15 minutes presentation, explanation or demonstration of a concept or a calculation, followed by a question displayed with a multiple choice. The students vote with their devices and the instructor shows the histogram of the answers. It is then asked to the students to reflect, talking with their peers during a few minutes, then vote again. The instructor can react to the results of the vote, deciding for example what kind of misconceptions he should comment on. 
The effectiveness of peer instruction was demonstrated by research [5]. We use it in a few courses, specifically in chemistry, mechanics, electrostatics and magnetostatics, with more to come. The implementation may be simple at the beginning, changing only by a small fraction the overall organisation of the course. The students are pleasantly surprised and become active during the lesson, asking questions and having the impression that their voice is heard. For the teacher, though it is unnecessary to change completely the lessons, peer instruction is a strong incentive to re-think a course and the associated pedagogy, making it a good introduction to flipped classrooms.

\subsection{Evaluation of the effectiveness of teaching}

Introducing some new techniques without evaluating their impact would lead in the short term to a failure, the teachers being less and less convinced of the relevance of those methods. Inventories, such as the Force Concept Inventory (FCI) [6] are tools used to assess the students learning gains. We tested the FCI on 60 students in 2014. The same test was proposed before the start of the course and after the end. The analysis is still unfinished, with no quantitative results at the time of the writing of this paper, though the trend seems to be the same as the one observed in the litterature. We intend to continue to use the FCI as well as other inventories such as CSEM [7] or BEMA [8].

\subsection{WIMS, an interactive online tool}

Among the many online exercizing tools that exist, we choose to use WIMS for the reasons explained hereafter. WIMS [9] [10] (Web Interactive Multipurpose Server) is a teaching platform containing interactive resources and tools to build them.

\subsubsection{WIMS philosophy}

WIMS was created by a network of teachers for their colleagues and for the students. There are exercises in mathematics, physics, chemistry, biology, french, electricity, electronics, english,... that go from an elementary school to university level. WIMS is open access and open source. There are more than 15 public servers in the world, more specifically in France, and one can create exercises on any public server, use them for a class without any registration process. If a user is nevertheless registered, he can share (but this is not compulsory) the exercises he produces with the community. The last important point is that WIMS allows for exercises with a deeply embedded random character, as well as an automatic correction process, even with formal answers.

\subsubsection{Pedagogical resources}

Making an exercise that includes some random data in the statement allows to present several times the same exercise to the student. It allows also to present the same exercise to two neighboring students without fear that they will look at each other's work. This simplifies the organisation of some evaluations. The automatic correction participates to this simplification.

There are various answer types, as for example :

- choice, with a click on a button or a picture

- association of elements with a drag and drop feature 
- numerical answer with a test for the precision

- formal, with an analysis of the answer. A simple formal engine is included

- clic on an image or on a 3D object, with the use of jSMol

- many other types, see the documentation

While building an exercise, a user can access some external software provided by the server, as jSMol, JSXGraph, povray, maxima, pari, octave and others.

\subsubsection{WIMS use}

We used WIMS for entry level tests in the first year of Licence, for the preparation and followup of tutorials, for assessment and self-assessment, mainly in mathematics and physics. We did not try to implement full exams, although WIMS is used for them in other universities.

\section{Results and students feedback}

Videos of physics courses received very positive feedbacks from all over the world.

Tutorials in small groups were also appreciated, $80 \%$ of the students being clearly more active and finding it more pleasant. However, the students often ask for a written solution, which in practice appear useless for most of them and is not very practical. This aspect is still under consideration.

Audience response devices and flipped classroom . The feedback is very positive here as well, the students are more active, attentive and concerned during the class, they ask more questions. While some research has proven the use of the devices effective, we still need to assess their effectiveness with various inventories. Peer instruction may be easily extended with flipped classrooms, which we tested during the first semester of 2014 in a course on Electrostatics and Magnetostatics. Before each lesson, the students were asked to prepare it by reading a few pages of notes on the subject, make a few simple exercises on the WIMS platform and ask questions on a specially formatted Moodle web page. This was twice as effective as asking the students to send an e-mail to the teacher. The exercises were done by around half of the students and there were, on average 25 questions asked per lesson, for a total of 40 students being effectively present during the class. The questions were answered during the lesson. The assessments were left similar to the ones of previous years and the histogram of the results (marks) showed a clear two bumps structure above (around the 12 mark) and below (around 7) the central mark (10 out of 20 in our case). The relative weight of the marks was similar to the one of the total number of students passing the exams over the number of students really present during the class, though we could not yet draw any firm conclusion based on those observations. A survey was made after the class and the students acknowledged having worked on average 50 minutes before each lesson and 30 minutes after. $85 \%$ of the students said that they understood the concepts better than in a course where they don't have to make any preparation beforehand. 


\section{Prospects and conclusions}

The actions described in this paper started as separate efforts to make the students progress, each teacher developing tools or methods that he felt were good to reach that goal. We convinced ourselves that whatever the tools, other teachers should be able to reuse them or to get some inspiration for the development of personal methods. Thus, our goal was to build a pedagogical toolkit in which there were enough different tools to align the teachers way of doing class and students way of learning (constructive alignment [1]). As an example, we used the toolkit in the Master's in Physics and Chemistry Education, asking students to look at videos or use WIMS, having tutorials in small groups or flipped classrooms.

The coordination is essential at a later stage, when several instructors begin to use the same tools, which is the case for example for the audience response devices, and for spreading the best practices. Clearly, we should continue our effort, particularly by extending the existing tools like videos of physics courses, WIMS online interactive exercises, tutorials in small groups, audience response devices or flipped classrooms, but also developing concept inventories to allow for a more precise evaluation of the effects of our efforts.

\section{References}

[1] J.B. Biggs and C. Tang, Teaching for Quality Learning at University (2011) McGraw-Hill and Open University Press, Maidenhead

[2] E. Mazur, Peer Instruction: A User's Manual Series in Educational Innovation (1997) Prentice Hall, Upper Saddle River, NJ

[3] E. Mazur, Peer Instruction: une méthode éprouvée d'enseignement interactif (2014) Presses polytechniques et universitaires romandes, Lausanne

[4] http://podcast.grenet.fr

[5] C. Crouch, E. Mazur, Peer Instruction: Ten Years of Experience and Results, Am. J. Phys., v69, 970-977 (2001)

[6] D. Hestenes, M. Wells, G. Swackhamer, Force Concept Inventory, The Physics Teacher, Vol. 30, March 1992, 141-158

[7] D. Maloney, T. O'Kuma, C. Hieggelke, A. Van Heuvelen, Surveying students conceptual knowledge of electricity and magnetism, Am. J. Phys. 69, S12 (2001)

[8] L. Ding, R. Chabay, B. Sherwood, and R. Beichner, Evaluating an electricity and magnetism assessment tool: Brief electricity and magnetism assessment, Phys. Rev. ST Phys. Educ. Res. 2, 010105 (2006)

[9] some WIMS servers : http://wims.univ-savoie.fr/wims/, https://wims.auto.u-psud.fr/wims/, http://wims.unice.fr/wims/

[10] The web site of "WIMSEDU", an association of teachers for the development of WIMS : http://wimsedu.info/ 